\title{
Structure Optimization of Phase-Coded Sounding Signals
}

\author{
D. SzczegielniaK ${ }^{a, *}$ AND A. Milewski ${ }^{b}$ \\ ${ }^{a}$ Institute of Telecommunications, University of Technology and Life Sciences \\ ul. Prof. S. Kaliskiego 7, 85-796 Bydgoszcz, Poland \\ ${ }^{b}$ Tele \& Radio Research Institute, Ratuszowa 11, 03-450 Warszawa, Poland
}

\begin{abstract}
Pulse compression with a small sidelobe level is one of challenges in designing of modern radar, sonar or ultrasound imaging systems. It may be performed by means of matched filter, that is by correlating the received sounding signal with a stored replica of the transmitted signal. The advantage of the pulse compression method is the increase of the average transmission power while retaining the range resolution corresponding to a short pulse. Phase coding is one of the early techniques for pulse compression of radar signals. Polyphase sequences, which have low sidelobe levels, ensure an easily detectable peak in the output of a matched filter, in other words an easy detection of a received sounding signal. In this paper, an evolutionary algorithm combined with a local optimizer is used to search for polyphase codes with a small sidelobe level of an aperiodic autocorrelation function. The evolutionary algorithm is based on a floating-point representation and the Gaussian mutation is used to produce offspring for the next generation. The self-adaptation mechanism is used to control the mutation operator during the evolutionary process. This research demonstrates that optimization methods can effectively find polyphase sequences with low autocorrelation and seems to be very promising for the future research in area of computer optimization for polyphase codes synthesis.
\end{abstract}

PACS numbers: 84.40.Ua, 84.40.Xb

\section{Introduction}

Pulse compression with a small sidelobe level is one of challenges in designing of modern radar, sonar or ultrasound imaging systems. It may be performed by means of matched filter, that is by correlating the received sounding signal with a stored replica of the transmitted signal. The advantage of the pulse compression method is the increase of the average transmission power while retaining the range resolution corresponding to a short pulse.

Phase coding is one of the early techniques for pulse compression of radar signals. Polyphase sequences, which have low sidelobe levels, ensure an easily detectable peak in the output of a matched filter, in other words an easy detection of a received sounding signal.

In this paper, an evolutionary algorithm combined with a local optimizer is used to search for polyphase codes with a small sidelobe level of an aperiodic autocorrelation function. The evolutionary algorithm is based on a floating-point representation and the Gaussian mutation is used to produce offspring for the next generation. The self-adaptation mechanism is used to control the mutation operator during the evolutionary process.

This research demonstrates that optimization methods can effectively find polyphase sequences with low autocorrelation and seems to be very promising for the future research in area of computer optimization for polyphase codes synthesis.

* corresponding author; e-mail: dszczeg@utp.edu.pl

\subsection{Problem definition}

The phase-coded pulse, shown in Fig. 1, is divided into $M$ bits of identical duration $t_{b}=T / M$, and each bit is assigned with a different phase value $\phi_{m}$. Such sequence of phase values is an example of a potential solution for the optimization problem of designing polyphase code.

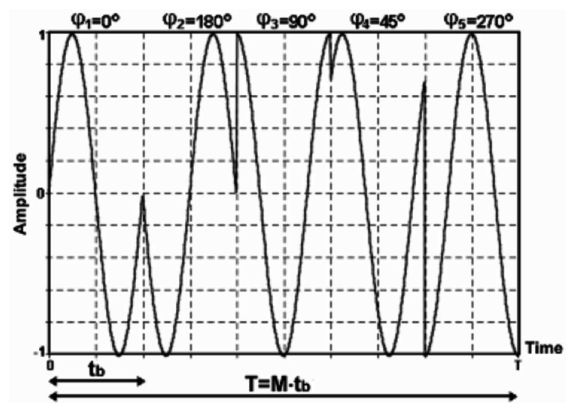

Fig. 1. An example of the phase-coded pulse.

The complex envelope of such phase-coded pulse is given by

$$
u(t)=\frac{1}{\sqrt{T}} \sum_{m=1}^{\mathrm{M}} \mathrm{e}^{j \varphi_{m}} \operatorname{rect}\left[\frac{t-(m-1) t_{b}}{t_{b}}\right] .
$$

The pulse compression goodness of a polyphase code is based on its autocorrelation function. The Autocorrelation function of phase-coded pulse is a continuous function of the delay $\tau$ and is defined by 


$$
R(\tau)=\int_{-\infty}^{\infty} u(t) u^{*}(t-\tau) \mathrm{d} t
$$

where the asterisk denotes the complex conjugate. This function may be expressed as the discrete aperiodic autocorrelation function according to the interpolation done in the complex plane and given by equation

$$
\begin{aligned}
& R(\tau)=R\left(k t_{b}+\eta\right) \\
& \quad=\frac{1}{t_{b} M}\left[\left(t_{b}-\eta\right) R[k]+\eta R[k+1]\right],
\end{aligned}
$$

where $R[k]$ is the discrete aperiodic autocorrelation function evaluated at $\tau=k$ and $0 \leq \eta<t_{b}$ [1]. An example of the autocorrelation function is shown in Fig. 2.

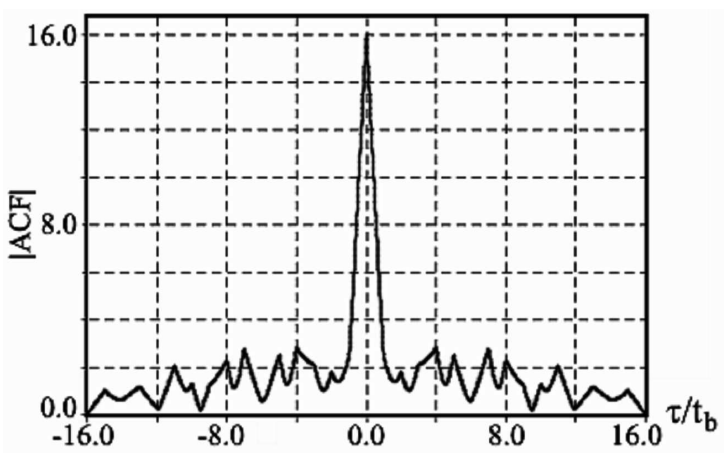

Fig. 2. An example of the autocorrelation function.

For examining the properties of polyphase sequences, it is sufficient to calculate the autocorrelation function only at integer multiples of the bit duration. The aperiodic autocorrelation coefficient $c_{k}$ may then be written as

$$
c_{k}=\sum_{m=1}^{\mathrm{M}-\mathrm{k}} a_{m} a_{m+k}^{*}, \quad k=-(M-1), \ldots,(M-1),
$$

where the asterisk denotes the complex conjugate and $a_{m}=\mathrm{e}^{j \varphi_{m}}$, for $1 \leq m \leq M, 0 \leq \phi_{m}<2 \pi$. Because the autocorrelation function is symmetrical with respect to its mainlobe $\left|c_{k}\right|=\left|-c_{k}\right|$, it may be rewritten with positive index $k$

$$
c_{k}=\sum_{m=1}^{\mathrm{M}-\mathrm{k}} \mathrm{e}^{j\left(\varphi_{m}-\varphi_{m+k}\right)}, \quad k=0, \ldots,(M-1) .
$$

A low autocorrelation for codes is usually described in terms of the maximum magnitude of its sidelobes level. Because first coefficient $\left|c_{0}\right|$ is the mainlobe and the last sidelobe equals one in any case $\left|c_{\mathrm{M}-1}\right|=1$, the objective function of the optimization problem can be eventually expressed as follows

$$
l_{\infty}(C)=\max \left\{\left|c_{k}\right|: 1 \leq k \leq M-2\right\} .
$$

Summing up, the optimization goal is to find a polyphase sequence which has a sidelobe level as low as it possibly can. The Problem under consideration is modeled as a nonlinear, NP-hard optimization problem in continuous variables and with numerous local optima [2].

\subsection{Effect of Doppler shifts}

The problem of finding polyphase sequences with increased resilience to Doppler shifts is very complicated and is based on examination of sidelobes in a three-dimensional ambiguity function. This function is widely used by radar designers as a means of examining different radar signals. It can provide insight about how different radar signals may be suitable for various radar applications. The ambiguity function may be defined as the complex-valued correlation between a waveform and a time-delayed, frequency-shifted replica of that waveform.

$$
\chi\left(\tau, f_{d}\right)=\int_{-\infty}^{\infty} u(t) u^{*}(t-\tau) \mathrm{e}^{j 2 \pi f_{d} t} \mathrm{~d} t
$$

Using the formula of the complex envelope of a phase-coded pulse in the definition of the ambiguity function gives:

$$
\begin{aligned}
& \chi\left(\tau, f_{d}\right)=\frac{1}{M} \sum_{n=0}^{\mathrm{M}-1} \sum_{m=0}^{\mathrm{M}-1} \mathrm{e}^{j 2 \pi f_{d} n t_{b}} \mathrm{e}^{j\left(\varphi_{n}-\varphi_{m}\right)} \\
& \quad \times \chi_{b}\left(\tau-(n-m) t_{b}, f_{d}\right)
\end{aligned}
$$

where $\chi_{b}\left(\tau, f_{d}\right)$ is the ambiguity function of each single-carrier subpulse. $\chi_{b}\left(\tau, f_{d}\right)$ is a triangular function along the delay axis in the interval $\pm t_{b}$ and a sinc function on the frequency shift axis [3].
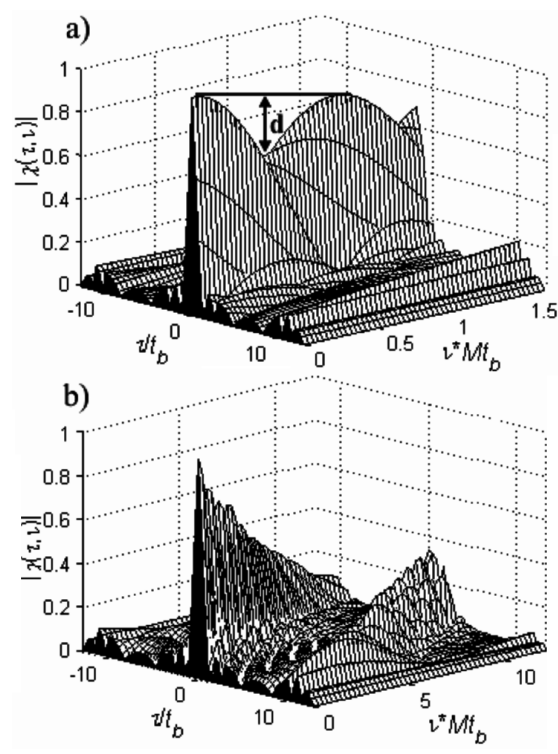

Fig. 3. Partial ambiguity functions for 16 element P4 code.

Doppler resilience is particularly essential in cases where long pulses are transmitted against high velocity targets. Once the received radar signal is Doppler shifted, the expected sidelobes are much higher than those predicted from observing only the autocorrelation function. Generally, polyphase codes are sensitive to Doppler shifts. Better Doppler tolerance of well-known polyphase codes, like P4 or Frank codes, results from 
that they were derived from the phase history of linear frequency-modulated or frequency-stepped pulses, which have a high resilience to Doppler effect. Figure 3 shows a partial ambiguity function for $\mathrm{P} 4$ code. It is worth noting that $\mathrm{P} 4$ code, similarly to other well-known polyphase codes, have a typical $4 \mathrm{~dB}$ cyclic loss of the correlation peak (marked by $d$ in Fig. 3a) with increasing Doppler frequency [4].

\section{Optimization algorithm}

In this research, an evolutionary algorithm combined with a local optimizer was used to search for polyphase codes with a small sidelobe level of an aperiodic autocorrelation function.

The optimization algorithm is shown in flowchart form in Fig. 4. The evolutionary algorithm begins by initializing a population of potential solutions for the objective function. Next the local optimizer is applied to improve starting points. New solutions are then created by mutating those of the initial population. All solutions then have their "fitness" evaluated and a selection criterion is applied to remove worse solutions. This process is iterated using the selected solutions until the stopping criterion is met.

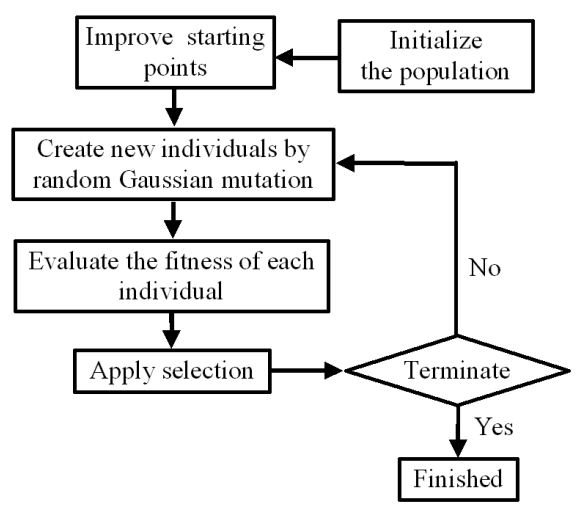

Fig. 4. The evolutionary algorithm in flowchart form.

For such nonlinear numerical optimization problem, the evolutionary algorithm was based on a floating-point representation. Each individual $x$ in the population was represented as a vector of floating-point numbers $x=\left(x_{1}\right.$, $\left.x_{2}, \ldots, x_{n}\right)$.

In order to produce offspring for the next generation, the Gaussian mutation was used. The crossover operator was rejected because of its disruptive influence on the convergence of the algorithm. Mutations were then realized by adding to each component of the vector a random Gaussian number with mean zero and standard deviation $\sigma$ changing during the evolutionary process and controlling by the self-adaptation mechanism. In this scheme, each individual has its own adaptable vector of $\sigma_{1}, \sigma_{2}$, $\ldots, \sigma_{n}$ values, which learns how to search the space of potential solutions. The mutations were realized according to formula

$$
\begin{aligned}
\sigma_{i}^{\prime} & =\sigma_{i} \mathrm{e}^{\mathrm{N}(0, \tau)}, \\
x_{i}^{\prime} & =x_{i}+N\left(0, \sigma_{i}^{\prime}\right),
\end{aligned}
$$

where $\tau$ is a parameter of the method that was set to $1 / \sqrt{n}$.

This mechanism implies a larger degree of freedom for adapting the search strategy to the topology of the fitness landscape [5].

For the selection of individuals for the next generation, tournament selection was applied. In this approach, the individuals in the population are randomly grouped in pairs, the fitness levels of two individuals are then compared to each other. The individual with the better fitness survives to the next iteration while the other is terminated.

The local optimizer was applied to find polyphase sequences which were good starting points for the evolutionary algorithm. Such approach significantly improved the performance of the evolutionary algorithm. The local method utilized another evaluation function than the evolutionary algorithm, which was the sum of the sidelobe energies but minima of both functions should lie close together.

$$
l_{2}(C)=\sqrt{\sum_{k=1}^{n-1}\left|c_{k}\right|^{2}}
$$

It is worth mentioning that the base energy function has more regular surface and is easier to optimize. Briefly speaking, the aim of the local optimizer, which utilized Eq. (11), was to move some initial points towards regions suspected of containing outstanding solutions for the latter function (defined by Eq. (6)).

The local optimizer was based on the Hooke-Jeeves direct search algorithm. This algorithm consists of two steps. First exploratory moves are made about a base point solution to determine an appropriate direction of search. Then, in second step - pattern search, the base point solution is moved, according to the previously determined direction, to a new location. If in exploratory search, all trials are not found the better value of the function, the algorithm goes back to the best recent base point and then step size is reduced and exploratory moves are made again. These stages are repeated until a step size becomes less than a pre-set value [7].

\section{Results}

The optimization method was tested for some arbitrary selected lengths from the range of 13 to 256 . The obtained results of this research are shown in Figs. 5 and 6 . In some cases, especially for shorter sequences, the algorithm found a polyphase Barker sequence occurring when a value of maximum sidelobe level is less or equal to one $[7,8]$. In general, such sequence might be regarded as a perfect solution. In the remaining cases, the results were also satisfactory.

The results are presented as values of Peak-to-Sidelobe Level (PSL) ratio, which is often used to quantify the 
performance of sidelobe suppression. PSL is simply expressed in decibels the ratio between the mainlobe and the highest sidelobe.

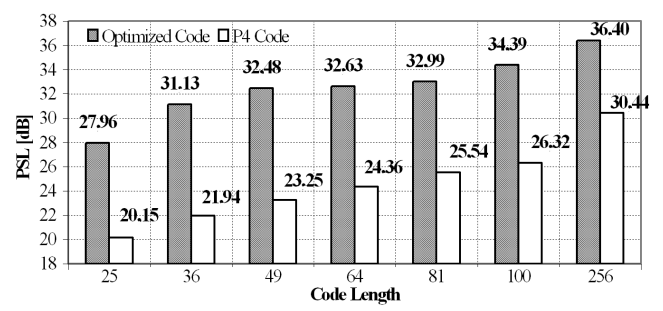

Fig. 5. The comparison of $\mathrm{P} 4$ sequences with codes obtained in the optimization process.

It is worth mentioning that the number of individuals in the population was ranging from one to three thousands and most of the results were obtained by only two or three runs of the algorithm therefore an influence of initial phase configurations on optimization results has not been studied yet.

It should be also mention that $\mathrm{P} 4$ codes have higher Doppler tolerance than obtained sequences in the optimization process without taking into consideration Doppler resilience. For example, the ambiguity function of a code obtained in this research with length 13 is shown in Fig. 6a. Generally, all sequences obtained in the optimization process without taking into consideration Doppler resilience have a similar shape to the ambiguity function shown in this figure. It can be easily noticed that the found polyphase code have a very poor Doppler tolerance, with the increasing Doppler frequency the mainlobe level decreases until at last it fades into the sidelobes.

The next example, taking into consideration Doppler shift in the optimization process is shown in Fig. $6 \mathrm{~b}$. In this case, the same hybrid optimization method, described in the paragraph 2 , with a suitably modified objective function was applied. This time the optimization method was searching the best properties of the correlation functions in given range of Doppler frequency. The obtained sequence is the outcome of initial research which demonstrates that the implemented optimization method can also search polyphase codes with increased resilience to Doppler shifts. The searching of phase-coded signals with higher Doppler tolerance is a very time consuming process therefore optimization of longer codes needs the growth of the computing power.

\section{Conclusions}

This research demonstrates that evolutionary strategies can effectively find polyphase sequences with the low autocorrelation and seems to be very promising for the future research in area of computer optimization for radar polyphase codes synthesis.
The growth of the computing power should bring much better results, especially for longer sequences, and allow
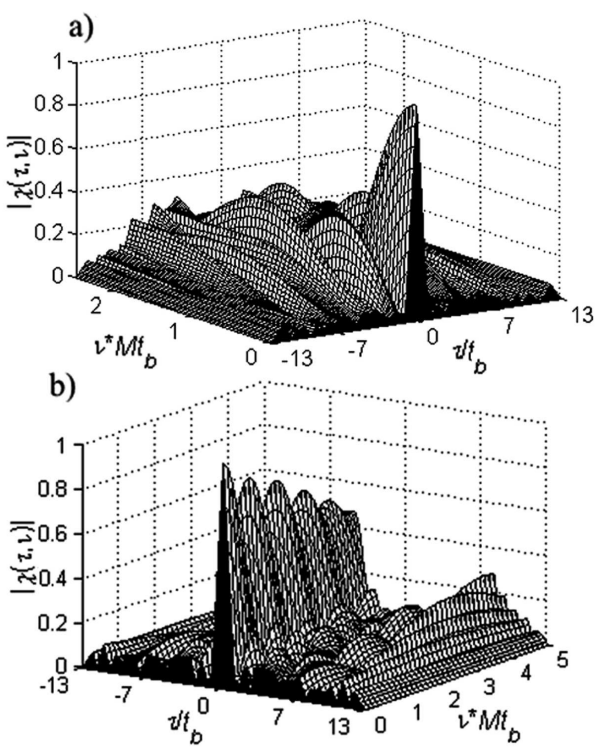

Fig. 6. Partial ambiguity functions of codes obtained in the optimization process (a) for 13 element code (PSL $=22.28 \mathrm{~dB}$ ); (b) for 13 element code with increased resilience to Doppler shift $(\mathrm{PSL}=18.55 \mathrm{~dB})$.

an optimization of longer codes with increased resilience to Doppler shifts. Therefore, future research will be oriented on implementation of parallel evolutionary algorithms using distributed programming.

In future work, the main emphasis will be placed on an improvement of spectral properties of phase-coded signals and resilience to Doppler shifts. The improvement of the evolutionary algorithm and the local optimizer will also be considered.

\section{References}

[1] N. Levanon, E. Mozeson, in: Radar Signals, John Wiley \& Sons, 2004, p. 100.

[2] N. Mladenović, J. Petrović, V. Kovacević-Vujcić, M. Cangalović, European Journal of Operations Research 151, 389 (2003).

[3] T. Misaridis, Ultrasound imaging using coded signals, Technical university of Denmark, 2001, p. 61.

[4] T. Felhauer, IEEE Trans. Aerosp. Electron. Syst., Vol. 30, 3, 869 (1994).

[5] Z. Michalewicz, D.B. Fogel, in: How to Solve It: Modern Heuristics, Springer Verlag, 2000, p. 276.

[6] W. Findeisen, J. Szymanowski, A. Wierzbicki, in: Theory and Computational Methods of Optimization, PWN, Warszawa 1977, p. 186, (in Polish).

[7] M. Friese, H. Zottmann, Electron. Lett. 30, 1996.

[8] P. Borwein, R. Ferguson, Information Theory, IEEE Transactions on Volume 51, 15642005. 